\title{
ELECTROMAGNETIC COMPATIBILITY DESIGN FOR KOMPSAT-2
}

\author{
Na-Young Lee ${ }^{\dagger}$, and Jin-Ho Lee \\ Korea Aerospace Research Institute, 45 Youseong, Daejon 305-333, Korea \\ E-mail: nylee@kari.re.kr, ljh@kari.re.kr
}

(Received September 19, 2003; Accepted November 13, 2003)

\begin{abstract}
It is quite essential that requirements allocation and analysis would be done for the electromagnetic compatibility (EMC) of all units when designing a satellite. Although KOMPSAT-2 inherits relatively large portions of the electrical designs from KOMPSAT-1, it has a new instrument and different combinations of sensors and actuators as well as their driving circuitry. Many requirements for the electromagnetic compatibility were modified and newly allocated for KOMPSAT-2. Naturally, they must be justified through analyses from the early stage of the program. In this paper, the EMC compatibility requirements for KOMPSAT2 are presented and verified for their suitability. In addition, some results, which were obtained from various analyses, are presented and discussed.
\end{abstract}

Keywords: KOMPSAT-2, electro-magnetic compatibility, conducted emission, conducted susceptibility, radiated emission, radiated susceptibility, spacecraft

\section{INTRODUCTION}

In the satellite design that consists of many different electrical units, it is important to define and establish the electromagnetic environment for the normal operation of each electrical unit. If one unit has stronger electromagnetic field radiation than the limit or causes more noise in interface lines than the level other units can tolerate, eventually there will be abnormal operation of certain unit or gradual degradation in the performance. In order to avoid such failure, specifications and requirements should be well defined from the beginning of the design phase. As design progresses, analyses and verifications would be necessarily performed to justify the requirements that were already defined. The electrical units will prove their compliance for the given requirement through the EMC test at unit level, and system level compliance will be demonstrated finally at the system level test after the integration (KARI 2002b).

The EMC of a satellite is mainly designed and tested according to the international standards such as MIL-STD-461E, but minimum modifications can be made when a unique hardware is used or special measures are required (KARI 2002b). KOMPSAT-1 had many modified or newly defined EMC requirements. Although KOMPSAT-2 directly inherits from KOMPSAT-1, there also have been many updates in system level EMC requirements because of the design changes and the different payload involved. The unit level EMC requirements, which did not exist in KOMPSAT-1, were newly allocated for the Koreanized units and the purchased items (KARI 2001a).

The EMC design and requirements allocations were performed for CE (Conducted Emission), CS (Conducted Susceptibility), RE (Radiated Emission) and RS (Radiated Susceptibility) (DOD

\footnotetext{
${ }^{\dagger}$ corresponding author
} 


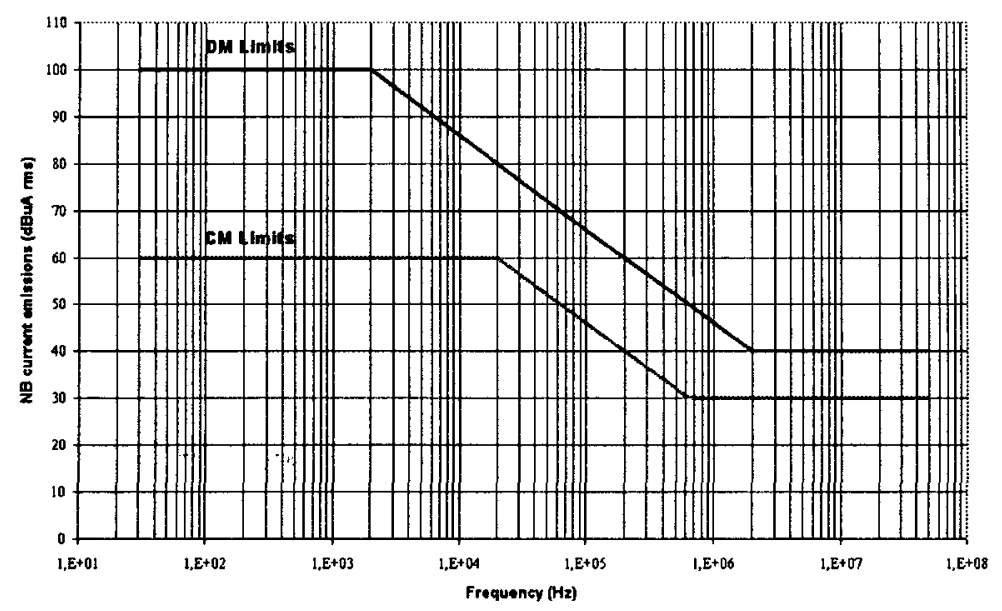

Figure 1. Conducted Emission Requirement for Satellite Units.

1999), and these items can also be divided into unit level, system level and the level of instrument, launch vehicle and ground equipments according to the defined target (KARI 2002b). In addition, the requirements for satellite grounding are defined. Satellite EMC design and verification additionally includes cautions required during test and integration process as well as the requirements for test, documentation and procedures for troubleshooting that will be performed when a problem occurs.

In this paper, the KOMPSAT-2 requirements for the major EMC characteristics are described, and the analysis results and the restrictions in design based on the requirements are presented and discussed.

\section{ELECTROMAGNETIC COMPATIBILITY REQUIREMENTS}

The EMC requirements for KOMPSAT-2 spacecraft are divided into unit level and system level. The unit level requirements shall define the specifications in detail and be applied to subcontractor units. The system level requirements outline overall design and test concepts that will be taken into account for the system integration.

The unit level requirements were allocated for $\mathrm{CE}, \mathrm{RE}$, specification for the grounding, shielding and bonding of the equipments as well as for ESD (Electro-Static Discharge) design (KARI 2001a). The requirements document also details the method of EMC tests that are necessary to achieve the compliances. Figure 1 shows the Conducted Emission (CE) limits that would be applied for differential mode and common mode respectively.

The system level requirements covering the whole KOMPSAT-2 satellite system include certain number of specific items not only for the spacecraft but also for the ground facility, payloads and the launch site environment. Additionally, the document provides the official process that should be followed when any non-conformance has been detected during the EMC design and verification process (KARI 2002b). Figure 2 shows the Radiated Susceptibility (RS) requirement for the satellite system with reflecting the payload and the launch site environments. 


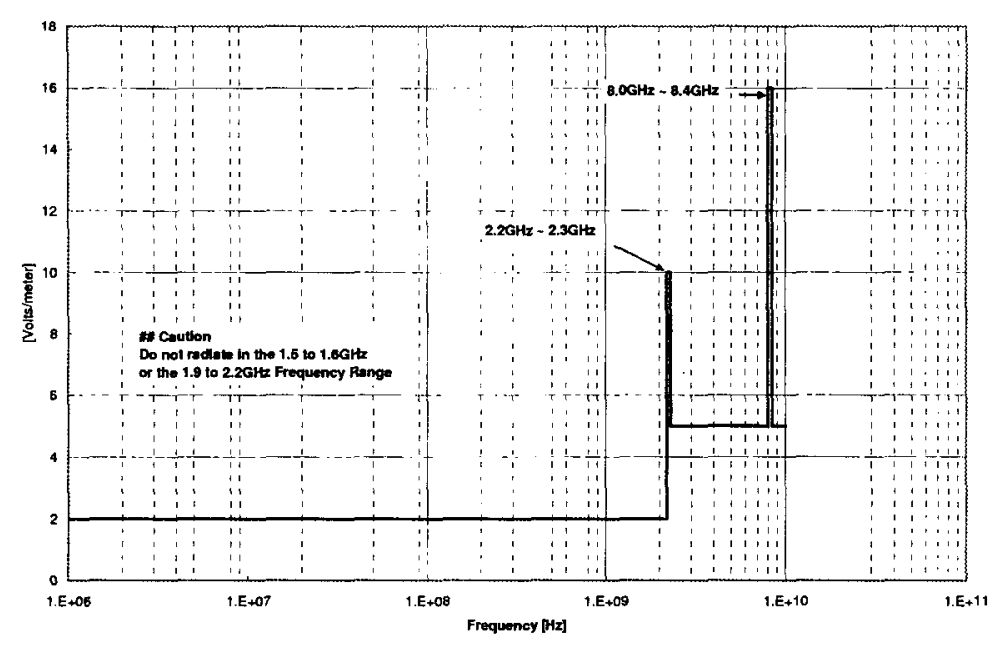

Figure 2. Radiated Susceptibility Requirement for Satellite System.

\section{CONDUCTION ANALYSIS}

\subsection{Transient analysis}

Transients concerned in satellite are as follows:

- Fuse Blow Transient

- Inrush Current

- Power Line Over-voltage

- Common Mode Transient

The fuse blow transient happens when a fuse blows due to the over currents that follows a short circuit of spacecraft equipment. If a short circuit happens, the bus voltage would drop to almost 0 [V] until the fuse blow. Just after the fuse blow, the bus voltage will shortly surge up to two times bigger than the normal voltage (KARI 2002c). A voltage curve varied from 0[V] to 64[V] would be used to demonstrate the compliance to this transient.

The inrush current of each unit causes the voltage fluctuations of the primary power lines and sometimes it influences seriously if the current scale is huge and the duration is long. The maximum inrush current of the spacecraft units was reported around 16[A] (KARI 2002c). Using the bus impedance requirement $(0.4[\Omega]$ ) (KARI 2002b), the voltage fiuctuation would reach maximum $7[\mathrm{~V}]$. Considering the voltage variance in orbit, the lowest voltage level was analyzed as 23.82[V] (KARI $2001 \mathrm{~b}$ ). The voltage under $20[\mathrm{~V}]$ could last for a few milliseconds in the worst case according to the calculation using $0.4[\Omega]$ bus impedance. However, the actual bus impedance was known to be slightly higher than $0.1[\Omega]$ by the analysis, so the voltage fluctuation by the inrush current would be only about $1[\mathrm{~V}]$.

The power line over-voltage can be produced from the radical change of the solar array power and it should be less than +42[V] (KARI 2002b). Figure 3 shows the voltage levels of PCU (Power Control Unit) board, outlet connector and main power bus when this transient happens. As shown in Figure 3, the transient voltage level was reduced in case of more loads. 


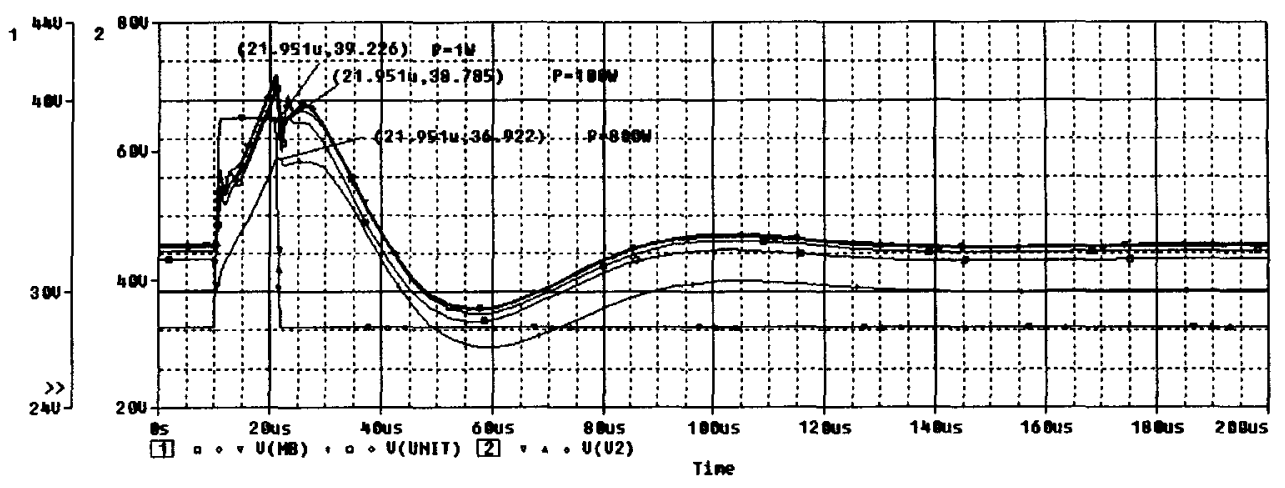

Figure 3. Voltage Variance at PCU (Power Supply Unit).

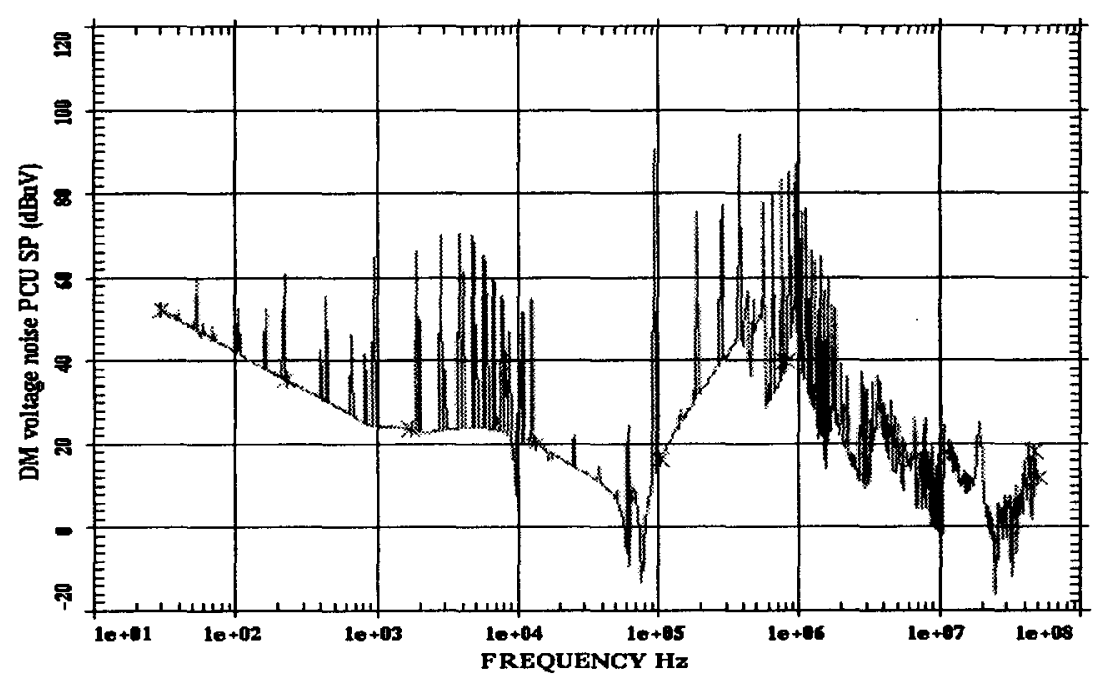

Figure 4. Noise Distribution on Primary Power Line.

In differential system like KOMPSAT-2, any load variance involves common mode transient, and that would impact on the secondary power of spacecraft units. The maximum amplitude of common mode spikes which possibly happen would never exceed 6[V] in any case, and it was experienced throughout the previous tests (KARI 2001a). The requirement was established for this common mode transient with more than $6 \mathrm{~dB}$ margins.

\subsection{Differential Voltage Noise in Primary and Secondary Power}

The voltage noises in the primary power could impact on all the spacecraft equipments, and therefore the influence level must be analyzed. A simulation was performed by using the impedance of the battery and PCU, and the noise model developed based on the unit test results. The noise and impedance models of units were also considered for the users of satellite primary power through the 


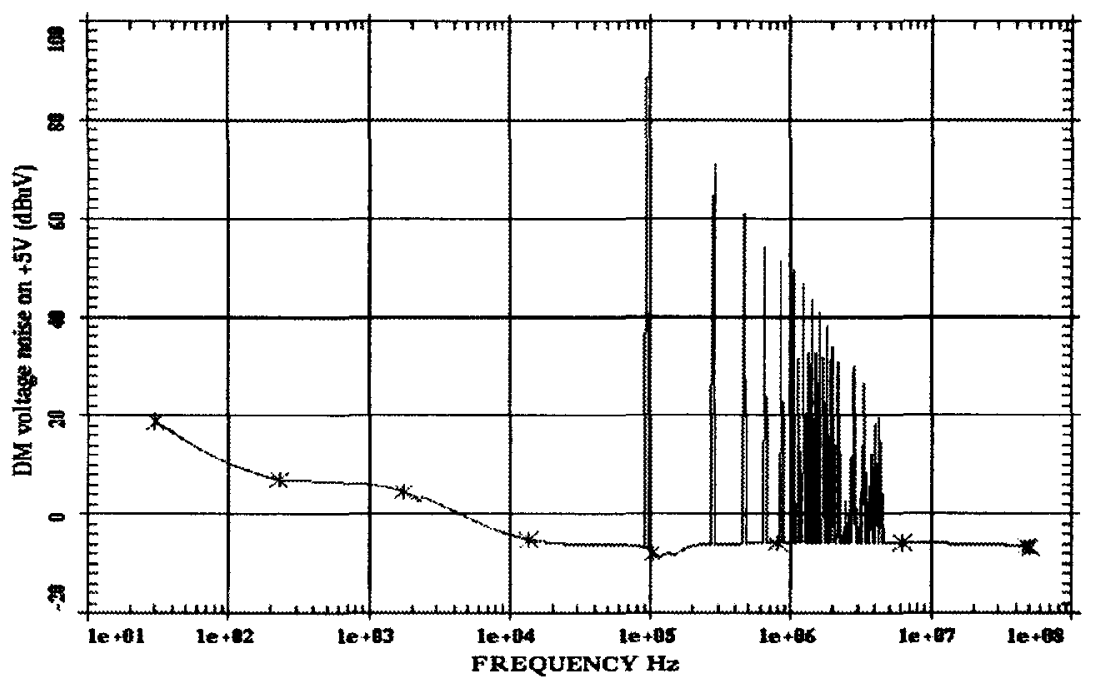

Figure 5. Noise Distribution on +5V PCU Star Point.

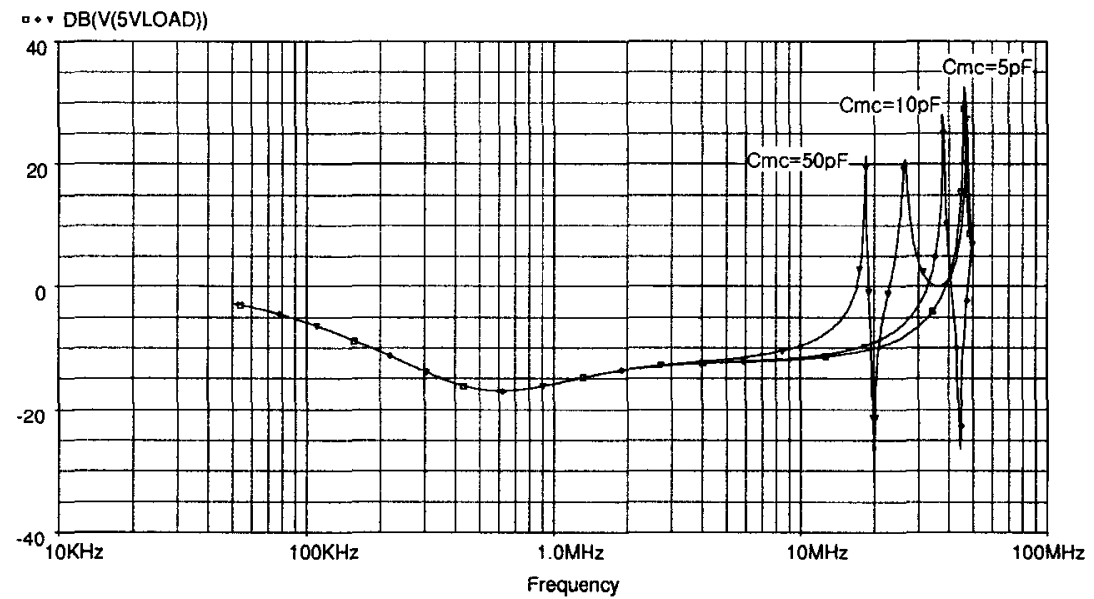

Figure 6. $+5 \mathrm{~V}$ Voltage Variance due to Satellite Structure Noise.

harness. Figure 4 presents the simulation result of differential noise distribution. From this result, it is understood that the noise of the GPS receiver and the star trackers are concentrated on near $100 \mathrm{~Hz}$ and the noise from the reaction wheel is dominant from $1 \mathrm{kHz}$ to $10 \mathrm{kHz}$ due to the current switching for wheel acceleration. Severe noises appeared at $94 \mathrm{kHz}$ and it was turned out that the power regulator switching of SAR (Solar Array Regulator) caused.

The secondary powers, i.e. $+15 \mathrm{~V},-15 \mathrm{~V}$ and $+5 \mathrm{~V}$ are converted from the primary power through the regulation of $\mathrm{PCU}$, so they are influenced by the regulation noise. The simulation for the $+5 \mathrm{~V}$ 
secondary power includes the impedance model of converter output, the noise model of PCU regulation under the assumption of the $150 \mathrm{Vpp}$ voltage ripple (KARI 2001 a), and the triangular wave shape at $94 \mathrm{kHz}$ switching frequency with considering the load impedance models of the units connected to $+5 \mathrm{~V}$ secondary power line. The model of the harness distribution between PCU and the loads were also implemented for an accurate analysis. The simulation result presents that the regulation of PCU is the major noise contributor from $94 \mathrm{kHz}$ to $10 \mathrm{MHz}$ range as shown in Figure 5.

\subsection{Satellite Structure Noise Effect}

KOMPSAT- 2 uses the structure grounding for secondary power therefore the secondary voltage of each unit could be affected by the noise flowing on the structure (KARI 2001a, KARI 2002b). In the simulation based on the circuit model and the impedance of PCU, the noise exceeding the allocated limit was detected in the secondary voltage when a noise source was applied to the structure ground (DOD 1999). Figure 6 is the result measured at the load side and it also involves the influence of the harness. The noise distributions existing at high frequency range depends on the stray capacitance value of the harness. To reduce the impact from the structure noise, the distance between the power supply lines and structure, as well as the harness length should be optimized.

\section{RADIATION ANALYSIS}

\subsection{Electrical Field Radiation Analysis in S-Band}

Any radiation in S-band could cause interference to the operation of the S-band receivers when they are receiving commands from ground. It is required to define permissible range, which should restrict the definition of units location. The S-band antennas are mounted on both nadir and the zenith platforms of spacecraft, but the nadir antenna is more vulnerable due to the relatively short distances from the units that possibly radiate in S-band. The analysis was performed with respect to the geometrical configuration as shown in Figure 7, and the antenna radiation patterns and the distance from the units would be very important factors. As depicted in Figure 7, S-band antenna is mounted on the top of the $30 \mathrm{~cm}$ boom and has a 118 off-line angle from the most influential unit that is located $0.75 \mathrm{~m}$ away. Considering the sensitivity of the receiver, the maximum permissible electrical field radiation would be $21 \mathrm{~dB} \mu \mathrm{V} / \mathrm{m}$ including $6 \mathrm{~dB}$ safety margin.

\subsection{Maximum Electrical Field Radiation Analysis in X-Band}

The payload of KOMPSAT-2 is different from that of KOMPSAT-1 and it has strong X-band RF power $(60 \mathrm{dBm}$ at maximum) (ELOP 2001). Therefore the other units should be adaptable to the increased electrical field radiation and be verified according to the modified requirements. First of all, it is required to derive the maximum radiation in X-band and then obtain the maximum offline angle. From these parameters, the radiation scale of the $\mathrm{X}$-band in certain distance can be calculated. Figure 8 depicts the geometrical relation between the satellite and ground station, which is used in this analysis. The maximum off-line angle $\theta^{\prime}$ would be $23^{\circ}$. With the antenna gain and the receiver output power at this angle, it is possible to compute the maximum electrical radiation. The compatibility to this radiation $(16 \mathrm{~V} / \mathrm{m})$ will be demonstrated through the unit or system level test.

\subsection{Radiated Coupling Analysis}

The radiated coupling analysis was performed to assess the system radiated emissions and to verify the risk of the interference coupling with the elements of a receiving chain such as S-Band receiver and GPS receiver. RF receivers must not lock on any unwanted radiated emissions that could be generated by an external device. Well known effect linked to the noise generation within the $R F$ receiver pass-bands is to decrease the signal to noise ratio out of allowable figures. Hence, a 


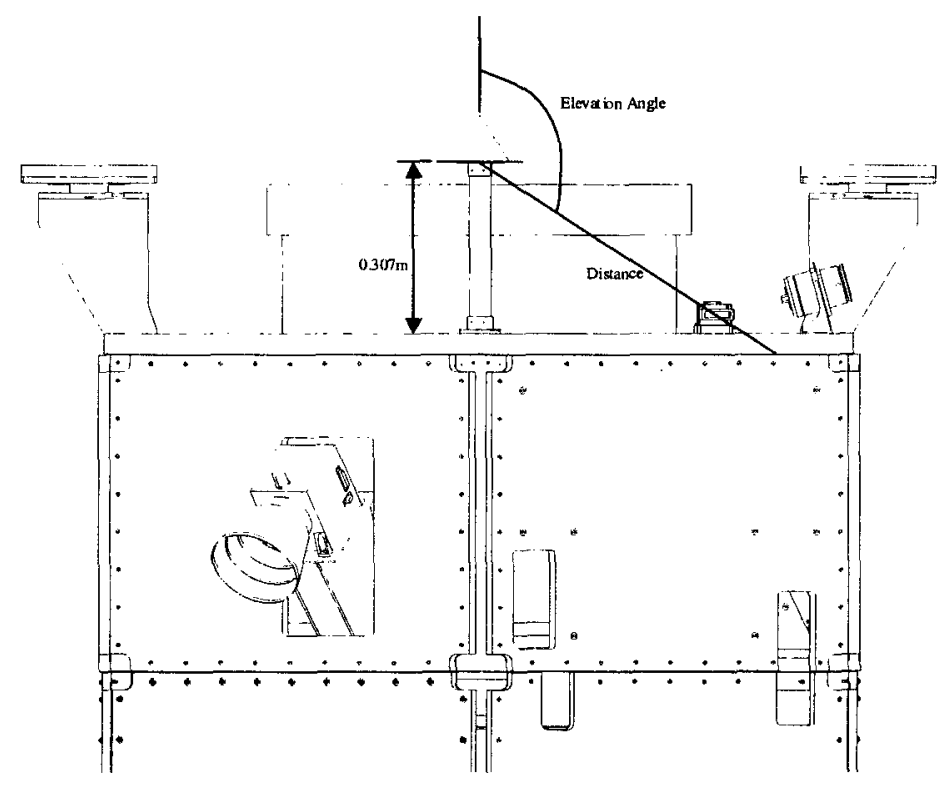

Figure 7. Nadir Platform Configuration with respect to S-band Antenna.

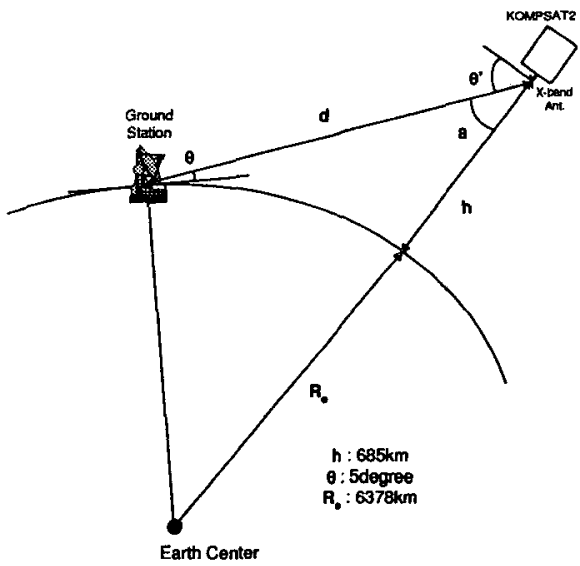

Figure 8. Geometrical Relations of Satellite and Ground Link.

specific attention should be paid to the noise contributors located outside the spacecraft cavity and near the receiving antenna. The star tracker is the most critical example.

The minimum distance between the star tracker and the GPS receiver is assessed to be $1.4 \mathrm{~m}$ in Figure 9. The angle between the direction of the source and the antenna axis is comprised between $135^{\circ}$ and $145^{\circ}$. From the minimum sensitivity threshold of the GPS antenna in L-band (KARI 


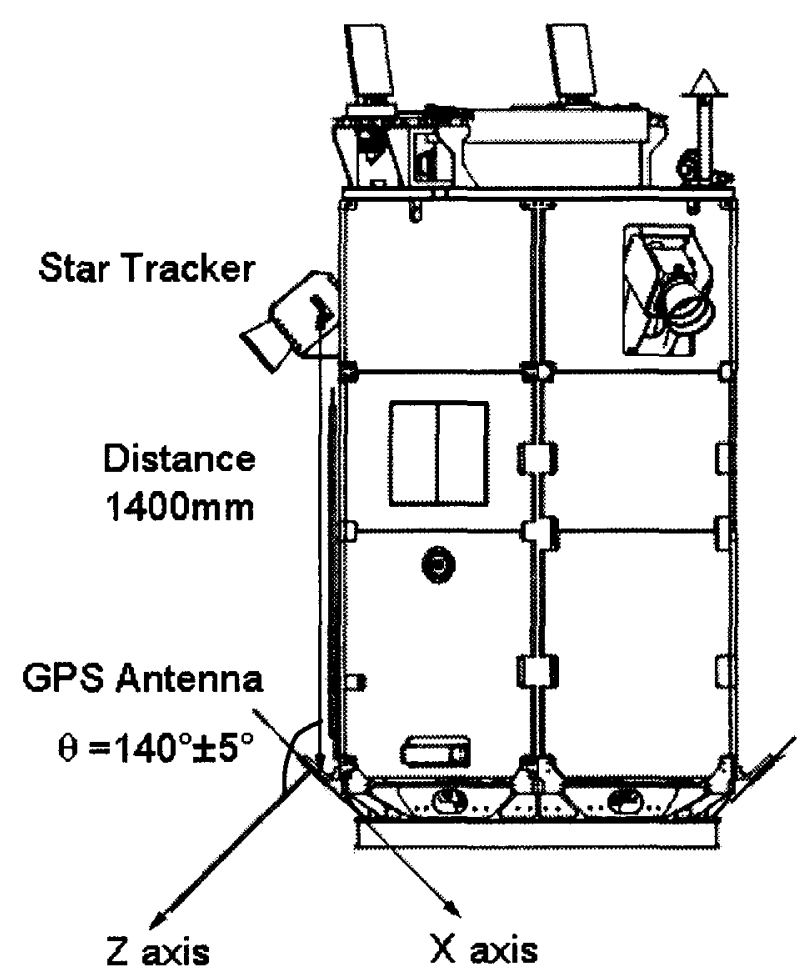

Figure 9. Coupling between Star Tracker and GPS Antenna.

2002a), the insertion losses of the RF cable and the antenna gain, the maximum allowable amplitude of the electrical field radiated emission in $\mathrm{L}$ band was calculated to $37.7 \mathrm{~dB} \mu \mathrm{V} / \mathrm{m}$.

\subsection{Avionics Structure Cavity Effects}

In a practical manner, the conditions of the propagation of the radiated electromagnetic wave inside the spacecraft cavity are different from the ones imposed by test conditions in anechoic chamber (assumed equivalent to free space propagation). For the regarded frequency range of the analysis [94kHz - $200 \mathrm{MHz}$ ], the shielded room inside the cavity might not be perfectly anechoic. The purpose of this analysis is to assess the cut-off frequency of the cavity when considering the dominant sources of radiation such as SAR and PCU power bundles. In addition, the order of magnitude of the electric field strength at different locations inside the cavity for several frequencies as well as the cavity insertion loss and the influence of the apertures would be identified through the analysis.

The first step consists of the definitions of the geometry and cavity materials, and mesh process; the number of meshes should comply and be suitable with regards to the frequencies that are to be analyzed. The modeling of the cable would be taken place to make a representative of radiating harness based on the definition and routing. Certain noise source would be applied to excite the simulation model and then eventually it would cause the current wave propagation on the cable. The amplitude of the current wave along the cable has been chosen to $1 \mathrm{Amp}$ for convenience. The condition of the current wave propagation along the cable must be transient wave; hence, the cable 


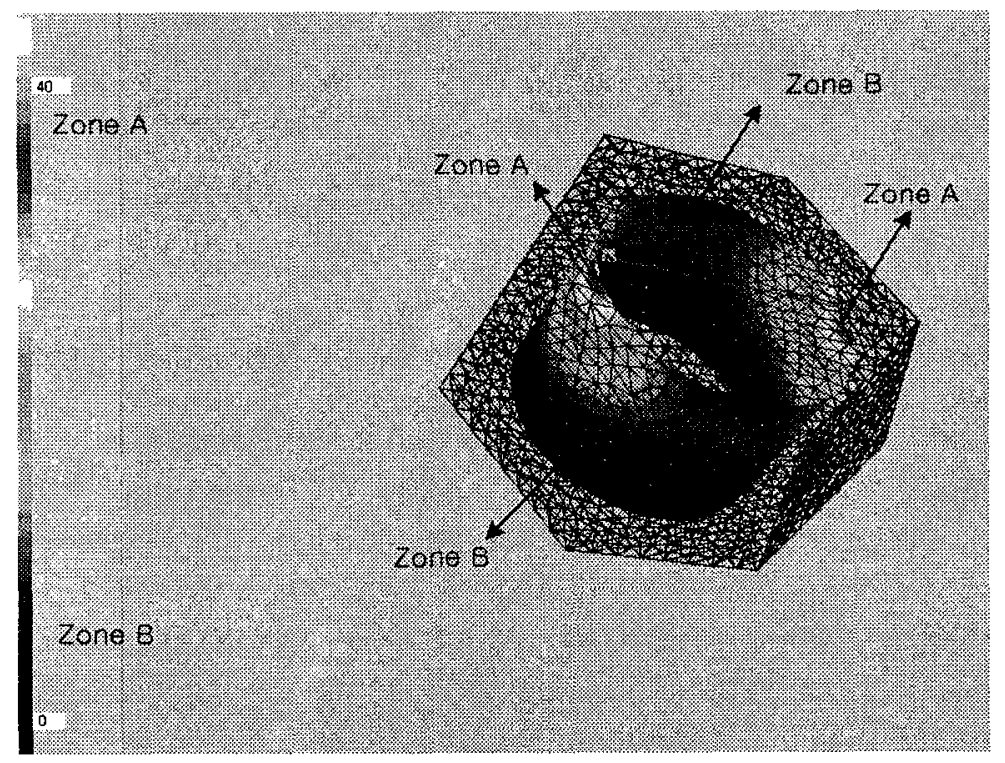

Figure 10. Electrical Field Levels inside Avionics Cavity at $94 \mathrm{kHz}$.

balanced impedance is assessed according to the bundle geometry. Finally, the current density and the $\mathrm{E}$ field levels on the different panels of the cavity structure would be obtained.

Figure 10 displays the simulation result over the electrical field inside the avionics cavity at 94 $\mathrm{kHz}$-SAR fundamental switching frequency. The scale was given in $\mathrm{dB}$; the zone A corresponds to the maximum level $30 \mathrm{~dB} \mu \mathrm{V} / \mathrm{m}$ for $0 \mathrm{~dB} \mu \mathrm{A}$ on the cable. By referring the measurements taken during the SAR EMC tests $(106 \mathrm{~dB} \mu \mathrm{V} / \mathrm{m})$, it leads the maximum field strength to $136 \mathrm{~dB} \mu \mathrm{V} / \mathrm{m}$ that is much higher than the susceptibility requirement for radiation (KARI 2002b). It should be noticed that this field value is limited to the area where the radiating cable ends. In real situation, the zone $\mathrm{B}$, which corresponds to the level of $20 \mathrm{~dB} \mu \mathrm{V} / \mathrm{m}$ for $0 \mathrm{~dB} \mu \mathrm{V} / \mathrm{m}$ on the cable, should be considered for other units. the field strength would then be down to less than $126 \mathrm{~dB} \mu \mathrm{V} / \mathrm{m}$, and is fully covered by the current requirement $(2 \mathrm{~V} / \mathrm{m})$. The simulation also showed that the first cut-off (resonating) frequency was $170 \mathrm{MHz}$.

\section{CONCLUSION}

This paper introduced KOMPSAT-2 requirements for the electromagnetic compatibility and analysis results. The analysis results and methods can be used for the EMC requirements allocation or troubleshooting in the test phase. In some area where test is not achievable, the analysis result itself can demonstrate the compliance to the given requirements. KOMPSAT- 2 system and units are now being tested for EMC based on the requirements and analysis results that were described in this paper, and this approach and method in EMC engineering will provide very useful lessons that are required in the development of a satellite system or other large scale electrical systems.

ACKNOWLEDGEMENTS: This study has been supported by Ministry of Science and Technology under the project of "Development of KOMPSAT-2." 


\section{REFERENCES}

DOD, 1999, Requirements for the control of EMI Characteristics of Subsystem and Equipment (Washington D.C: Department of Defense), MIL-STD-461E

ELOP, 2001, MSC CDR Data Package (Israel: Electro-Optics Industries LTD.)

KARI, 2001a, Requirements for the Units EMC Design, Performance and Tests (Daejeon: Korea Aerospace Research Institute), K2-SP-410-005

KARI, 2001b, KOMPSAT-2 Power Transient Analysis Report (Daejeon: Korea Aerospace Research Institute), K2- KS-RP-0114

KARI, 2002a, KOMPSAT2 Radiated EMC analysis (Daejeon: Korea Aerospace Research Institute), K2-NT-0127-T-ASTR

KARI, 2002b, Electromagnetic Compatibility and Control Plan (Daejeon: Korea Aerospace Research Institute), K2-SP-330-003

KARI, 2002c, KOMPSAT2 EDI CDA data package (Daejeon: Korea Aerospace Research Institute) 